\title{
Coherent combination of ultrafast fiber amplifiers
}

\author{
Marc Hanna $^{1}$, Florent Guichard ${ }^{1,2}$, Yoann Zaouter ${ }^{2}$, \\ Dimitris N Papadopoulos ${ }^{3}$, Frédéric Druon ${ }^{1}$ and Patrick Georges ${ }^{1}$ \\ ${ }^{1}$ Laboratoire Charles Fabry, UMR 8501, Institut d'Optique, CNRS, Univ. Paris-Sud, Université Paris- \\ Saclay, 2 Av. A. Fresnel, 91127 Palaiseau, France \\ ${ }^{2}$ Amplitude Systèmes, 11 avenue de Canteranne, Cité de la Photonique, 33600, Pessac, France \\ ${ }^{3}$ Laboratoire d'Utilisation des Lasers Intenses, Ecole Polytechnique, CNRS, CEA, UPMC, Route de \\ Saclay, 91128 Palaiseau, France
}

E-mail: marc.hanna@institutoptique.fr

\begin{abstract}
We review recent progress in coherent combining of femtosecond pulses amplified in optical fibers as a way to scale the peak and average power of ultrafast sources. Different methods of achieving coherent pulse addition in space (beam combining) and time (divided pulse amplification) domains are described. These architectures can be widely classified into active methods, where the relative phases between pulses are subject to a servomechanism, and passive methods, where phase matching is inherent to the geometry. Other experiments that combine pulses with different spectral contents, pulses that have been nonlinearly broadened or successive pulses from a mode-locked laser oscillator, are then presented. All these techniques allow access to unprecedented parameter range for fiber ultrafast sources.
\end{abstract}

Keywords: coherent combining, ultrafast optics, fiber amplifiers

\section{Introduction}

Fiber amplifiers have become a major technology in the high average power laser sources landscape (Richardson et al 2010). They combine numerous advantages making them ideally suited for an increasing number of applications, including industrial material processing, medical treatment and scientific experiments. The double clad geometry allows pumping with high power laser diode systems, and provides excellent power transfer efficiency from the pump to the single transverse mode output beam, determined by the waveguide properties of the fiber core. Ytterbium-doped fibers exhibit a very low quantum defect from the pump at $976 \mathrm{~nm}$ to the signal around $1030 \mathrm{~nm}$, yielding high opticalto-optical efficiency and low heat deposition in the gain medium. The large surface to volume ratio of the fiber, related to its geometrical shape, facilitates heat dissipation. These unique properties have allowed the design of multi-kW continuous wave $(\mathrm{CW})$ diffraction-limited fiber laser sources.

When used to amplify femtosecond pulses, at large peak power levels, the peculiar geometry of fiber amplifiers turns into a disadvantage in terms of allowable pulse energy when compared to other gain medium shapes. The optical mode is strongly confined within the fiber core, and guided over metersized lengths, enhancing nonlinear interaction with the fiber material. Effects such as self-phase modulation (SPM), selffocusing, optical damage at the facets, appear at modest pulse energy levels compared to typical bulk amplifiers. Moreover, the limited gain bandwidth of ytterbium-doped glass, combined with typical large gain levels, induce gain narrowing and limit the achievable amplified pulsewidth to $300-500 \mathrm{fs}$.

To overcome these drawbacks of ultrafast fiber amplifiers, the main idea is to reduce the optical intensity inside the fiber core by distributing the power over time and space. 
Chirped-pulse amplification (CPA) consists in introducing a large amount of group-velocity dispersion (GVD) to stretch the input pulse in time by a large ratio, thereby decreasing the peak power by the same ratio (Strickland and Mourou 1985). At the output of the amplifier, pulses are compressed down to their initial duration by introducing the opposite GVD. The stretched pulse duration is limited to a few nanoseconds by the size and cost of optical gratings that are used in stretchers and compressors. In space, specific quasi-singlemode fibers with large mode area have been designed and fabricated to reduce nonlinear effects along amplification (Stutzki et al 2014). Scaling of the mode area is limited by the appearance of higher order guided modes that decrease the output beam quality and the temporal contrast of the pulses. For Ytterbium-doped fibers, the current maximum achievable mode-field diameter is about $100 \mu \mathrm{m}$. These techniques have been developed over the last twenty years, allowing the generation of $\mathrm{mJ}$ femtosecond pulses (Eidam et al 2011). However, fibers that are used to reach these energy levels are very large mode area rodtype fibers in which thermo-optical effects limit the average power to around $100 \mathrm{~W}$.

To further scale the peak and average power of femtosecond fiber amplifiers, it is a natural idea to extend the distribution of power to several beams in space and pulses in the time domain, leading to the concept of coherent combining. Coherent beam combining has been first implemented for $\mathrm{CW}$ lasers (Fan 2005), before being extended to the pulsed regime for nanosecond (Cheung et al 2008a, Lombard et al 2011) and femtosecond pulses. The main idea is to split an initial pulse to be amplified in several replicas either in multiple beams (coherent beam combining) or at multiple delays (divided pulse architecture), proceed to amplification, then combine them by controlling the relative optical phase between all replicas. Most of the experiments in the field have been performed with fiber amplifiers because of their potential for integration and high average power operation, and because beam quality is an essential feature to be able to efficiently combine several beams. First demonstrations appeared in 2010-2011 (Seise et al 2010, Daniault et al 2011a), showing active beam combining of two femtosecond fiber amplifiers. Since then, a large number of architectures involving active or passive phase stabilization in the space and time domains have been proposed and demonstrated. These concepts have already allowed establishing new record performances for fiber amplifiers, with multi-mJ sources emitting an average power of several hundred watts (Klenke et al 2014a).

In this review, we review the concepts that have been introduced over the last few years, from general notions to the description of specific laser source architectures. The discussion is extended outside the scope of fiber amplifiers to describe the use of the same femtosecond coherent combining techniques to scale pulse energy in nonlinear temporal postcompression waveguide setups, demonstrating the use of these concepts for pulse durations down to the few-cycle regime. Femtosecond resonant passive enhancement cavities are then described as a different type of coherent combining strategy. To conclude, large scale femtosecond laser facility

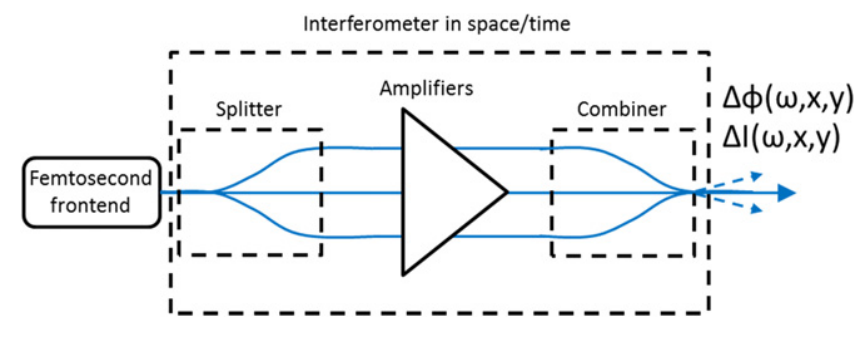

Figure 1. Layout of a general coherent combining system.

proposals based on coherent combining are shortly described. Indeed, coherent combining techniques constitute a very promising path towards future high energy, high repetition rate ultrafast sources for applications such as particle acceleration, and high power secondary sources of extreme ultraviolet radiation and $\mathrm{x}$-rays.

\section{General notions}

We first describe general aspects of femtosecond coherent combining systems. After exposing the basic principle, various implementations of splitting and combining setups are considered. Phase noise properties of fiber amplifiers are then introduced, and various techniques used to lock the optical phase of several pulses are explained. Finally, the most common performance metric of a coherent combining system, denoted as combining efficiency, is presented.

\subsection{Coherent addition of short pulse electric fields}

Experimental setups used to perform coherent combination of femtosecond pulses can all be seen as optical interferometers that include amplifiers in the multiple possible paths, as depicted in figure 1. An initial femtosecond frontend is used to generate the optical pulses to be amplified. These pulses are subsequently split and sent in the interferometer arms, amplified, and recombined in a single output pulse. The efficiency of the system is related to the ability to control the phase accumulated by optical pulses in each path in order to guarantee constructive interference on one of the interferometer outputs. This constructive interference condition must be satisfied over both the space/wavevector (Goodno et al 2010) and time/spectral domains (Klenke et al 2011, Hanna et al 2013). In practice, and given the fact that most coherent combining setups are optimized to combine identical beams and pulses, this condition implies that the beams to be combined should exhibit the same spatial profile and wavefront, together with the same spectral content and spectral phase. The latter condition is specific to ultrafast coherent combining systems, and can be seen intuitively in the time domain: in addition to the phase matching condition of optical carriers, the pulses to be combined must arrive on the combining element at the same time or group delay, which is determined by the first-order Taylor expansion of the optical phase with respect to angular frequency. Higher order terms in this expansion determine the temporal profile of the pulses, 


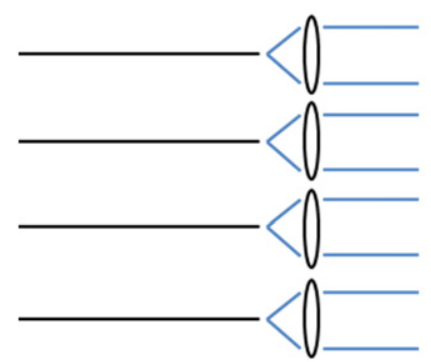

Tiled aperture

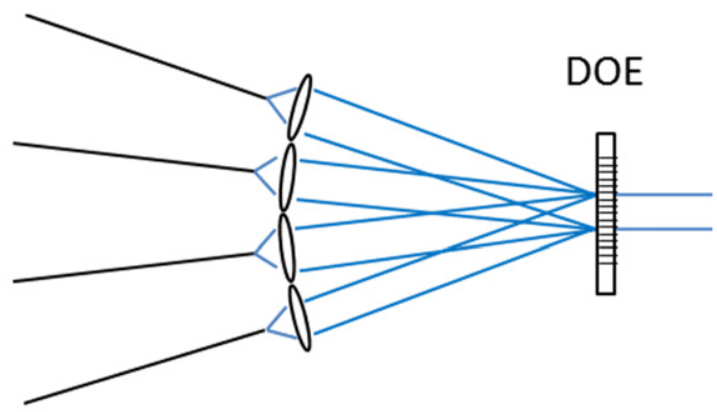

Filled aperture

Figure 2. Tiled aperture and filled aperture beam combining geometries.

and must also be matched. Possible optical phase mismatch of the beam/pulses to be combined translates into a decrease of the combining efficiency, and therefore leads to significant power loss into unused output ports of the interferometer and/ or decrease of the combined peak power as detailed in section 2.5 .

\subsection{Splitters and combiners in space and time}

Fabrication of the amplifying interferometer necessitates the use of optical components to perform the dividing and combining functions. Dividing and combining can in principle be done using the same components, but can differ in practice due to various considerations, in particular power handling: the combiner is a critical component that must be able to withstand the final average power and peak intensity without introducing losses.

Coherent beam combining geometries can be classified in two broad categories, denoted as filled aperture and tiled aperture, as illustrated in figure 2. In the tiled aperture geometry, the beams to be combined are simply positioned next to one another in the near field. Coherent combining is effective only in the far field, where a single spatial lobe is synthesized if the optical phase of the sub-beams is controlled. In this case, the combiner element can be either a simple lens or even just the diffraction over a certain distance. This simplicity makes the tiled aperture architecture attractive when the number of beams to be combined is large, or when power handling issues are important. The spatial properties of the combined beam depend on the precise geometrical arrangement. In particular, the filling factor of the composite beam determines the fraction of power contained in the central combined lobe. The combined beam properties also depend on the phase relationship between sub-beams. If a large number of beams are combined, control of this phase relationship could enable the control of the combined beam, for instance beam steering capabilities. In the framework of femtosecond beam combining systems, only one experiment reports the use of the tiled aperture geometry to our knowledge (Ramirez et al 2015). This experiment highlights one characteristic of the tiled aperture geometry for ultrafast pulses that was previously theoretically identified (Hanna et al 2013): residual group velocity mismatch between sub- beams are translated to spatio-temporal couplings in the output beam.

The filled aperture approach is closer to the idea of the interferometer, since the beams corresponding to each interferometer path are superposed onto a combining element to generate a single output beam. In this case, and if coherent addition is achieved ideally, the spatial properties of the output beam are the same as each individual beam before combination. Almost all femtosecond beam combining experiments use this approach, because the number of combined beams is less than 10 in most cases, and because the generated output beam generally exhibits better spatial quality. The simplest way to divide/combine beams in this case is to use a two-port component such as beamsplitters (Daniault et al 2011a) or polarizing beamsplitters (Seise et al 2010). Cascading such binary components allows in principle the generation and combination of an arbitrary number of beams, although component losses are accumulated in the process.

To combine a large number of beams with a single component using the filled aperture combining geometry, diffractive optical elements (DOE) have been used in CW combining experiments (Cheung et al 2008b). A DOE is essentially a phase grating that is optimized to split an incident beam into $\mathrm{N}$ output beams with a large efficiency and roughly equal power in each beam. The relative phase relationship of the $\mathrm{N}$ output beams is deterministic and fixed by grating design. Due to the reciprocal nature of light propagation, this component can also be used backwards: if $\mathrm{N}$ beams are incident on the DOE with angles that correspond to optimized diffraction orders, and if their phase relationship matches the DOE design, the output power is mostly carried by a single beam. DOEs have been demonstrated both in one dimensional (Redmond et al 2012) and two dimensional designs (Thielen et al 2012), with large efficiencies and at high power $(\mathrm{kW})$ level. In the femtosecond regime, the large optical bandwidth of pulses to be combined can become incompatible with the use of DOEs: like any grating, DOEs introduce angular dispersion, leading to a recombined beam that carries angular chirp. This effect, together with the modest number of combined beams in reported work, has prevented an experimental demonstration of the use of DOEs in a femtosecond beam combining experiment. However, depending on the optical bandwidth and the DOE design, this effect can be negligible. Optical 


\section{Birefringent crystals}

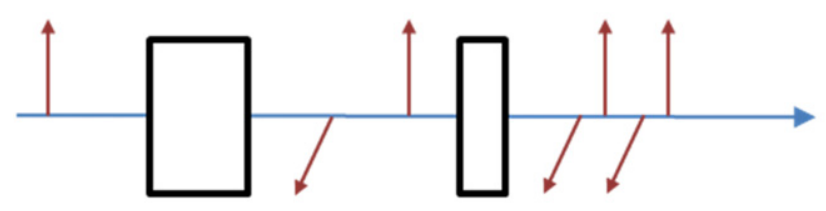

Freespace delay lines

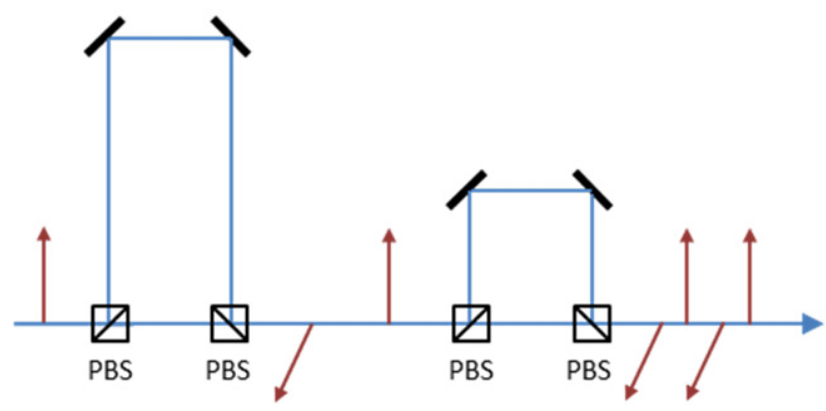

Figure 3. Splitters and combiners in the time domain. The red arrows represent the polarization state of successive pulses. Waveplates have been omitted. PBS: polarizing beam splitters.

arrangements have also been proposed to reduce this effect (Zapata-Rodríguez and Caballero 2007), and it can also be preor post-compensated using arrangements of complementary angularly dispersive components.

Splitter and combiner subsystems are also needed to generate several replicas of an initial pulse in the time domain. They use the polarization state of the light to route replicas of the pulse through the time domain interferometer. For values of group delay between replicas up to a few tens of picoseconds, crystals that exhibit a large group birefringence can be used to separate the ordinary and extraordinary components of incident light, as shown in figure 3 (Zhou et al 2007). Such crystals include yttrium vanadate (Kong et al 2012) and calcite (Jacqmin et al 2015). These components allow an inline interferometer configuration. When larger delays are required, for example to separate pulses that are stretched to nanosecond durations in CPA amplifiers, freespace delay lines (Zaouter et al 2013) can be used in conjunction with polarizers to fabricate unbalanced MachZehnder interferometer that are equivalent to birefringent crystals, as shown in figure 3 . In this case, a careful and stable alignment of the interferometer must be done to guarantee a single beam at the output. Both implementations can be used to split and/or to combine pulses in time.

\subsection{Phase noise in fiber amplifiers}

In beam combining experiments, since a single femtosecond oscillator is used to seed several fiber amplifiers, phase fluctuations between beams to be combined originate solely from fiber amplifiers. It is therefore important to measure and analyze phase noise introduced by such amplifiers, for instance to determine the characteristics of the feedback loop in terms of bandwidth and gain in active beam combining systems. Such studies were carried out in the frame of $\mathrm{CW}$ coherent combining (Augst et al 2004) by performing self-heterodyning experiments between a reference beam and a fiber amplified beam. These experiments show that phase fluctuations are dominated by environmental thermal and acoustic contributions, and become negligible beyond $10 \mathrm{kHz}$. More specifically, there is typically a large and monotonic change of the optical phase over several thousand waves at amplifier turn-on, corresponding to heat up time. During this time, the group delay also continuously drifts over several hundred femtoseconds. Once the thermal steady-state is reached, the optical phase varies over a typical scale of $\pi / 10$ on a millisecond time scale. Over longer time intervals of tens of seconds, the relative phase excursion varies over a typical range of tens of radians that can be reduced to a few radians by carefully isolating the systems acoustically. These numbers correspond to laboratory environments, and since the origin of the noise is mostly thermal and acoustic, they can vastly differ depending on experimental conditions. Feedback loops that control the relative phase between amplifiers usually feature bandwidths ranging from $100 \mathrm{~Hz}$ to $10 \mathrm{kHz}$, allowing the use of various phase actuators such as piezo-mounted mirrors, electro-optic integrated phase modulators, and spatial light modulators.

Because of the mostly environmental nature of the phase noise, it is expected that integrating the multiple fiber amplifiers into a single structure through the use of a multicore fiber should drastically reduce phase fluctuations between beams to be combined. This was verified in various multicore amplifier experiments (Hartl et al 2009), and a recently reported work shows active combining in the femtosecond regime with a decoupled 7-core fiber using a feedback bandwidth of only $2 \mathrm{~Hz}$ (Ramirez et al 2015).

For ultrafast combining experiments, the group delay introduced by each amplifier and higher orders of the spectral phase could in principle also fluctuate in time. However, in reported femtosecond experiments, only a static manual adjustment of the relative group delay is performed, and the systems do not exhibit large fluctuations of delay between interferometer arms once the thermal steady state is reached. It is expected that combining systems should be increasingly sensitive to such fluctuations when the pulses to be combined are shorter, or equivalently the optical bandwidth larger. In this case, a group delay locking mechanism, as demonstrated in (Weiss et al 2012), might be implemented. It simply consists in achieving simultaneous phase locking at two well separated wavelength components, thereby reducing the spectral phase slope, i. e. the group delay, to zero.

\subsection{Phase measurement and active feedback techniques}

Active beam combining of femtosecond fiber amplifiers requires real-time feedback on the relative optical phase between beams. This feedback is based on the detection of one or several control signals from the output of the system. Phase control systems can be broadly classified in two categories: phase control is either based on a deterministic measurement of relative phases between beams, or on stochastic 

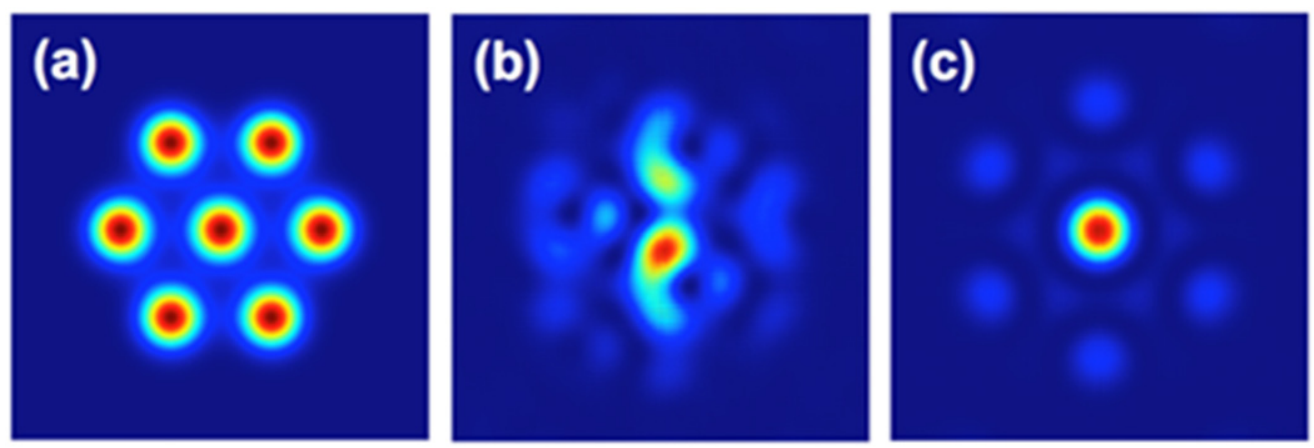

Figure 4. Example of a tiled aperture system obtained with numerical simulations. (a) Tiled beam in hexagonal arrangement. (b) Combined beam in the case of random phases for the sub-apertures. (c) Ideal combined beam. Adapted from (Ramirez et al 2015).

hill-climbing algorithms that seek to maximize a metric of the system performance.

Among systems based on phase measurement, several techniques have been implemented and can be used for ultrafast fiber amplifiers. Frequency tagging, or LOCSET (locking of optical coherence via single-detector electronicfrequency tagging) (Shay et al 2007), consists in introducing a small-amplitude phase modulation on each beam at a predetermined frequency. Heterodyne detection of this signal with either a reference signal or an unmodulated beam (denoted as self-referenced LOCSET), followed by lock-in amplification, allow identifying and measuring the relative optical phase of each beam with a single detector at the output of the system. These phases are then used as error signals by the feedback system to generate control signals applied on phase actuators located in each interferometer arm. The nonzero amplitude of phase dithers induces a residual phase error that has in most practical case negligible impact on the combining efficiency. In CW regime LOCSET can be easily scaled to a large number of channels and has been used to combine 32 low-power CW laser beams (Flores et al 2013). In pulsed regime, the applied phase dither frequency must be less than half the repetition rate of the system. For high energy systems, repetition rates can be in the range $10 \mathrm{kHz}-$ $1 \mathrm{MHz}$, and scaling the number of channels is the result of a tradeoff between number of channels and feedback bandwidth. Due to its simplicity of use and efficiency, frequency tagging has been used in several femtosecond fiber amplifiers combining experiments (Siiman et al 2012, Daniault et al 2011a, Kienel et al 2015).

For coherent combining systems that use two-port polarizing beam splitters as combining components, measuring the relative phase between beams is equivalent to measuring the output polarization state. In this case, the method introduced by Hänsch and Couillaud (Hänsch and Couillaud 1980) can be used to generate an error signal that allows phase locking. This technique requires the use of $\mathrm{N}-1$ detectors, where $\mathrm{N}$ is the number of beams to be combined. It has been implemented in femtosecond systems (Seise et al 2010).

In the case of tiled aperture systems, optical phase measurement can also be done in space by measuring the position of interference fringes between beams to be combined or with respect to a reference beam. In particular, lateral shearing interferometry can be used to measure relative phases with a single camera for a large number of channels and with $\mathrm{kHz}$ bandwidths (Bourderionnet et al 2011).

The second broad category of phase control system used in the frame of coherent combining is based on algorithms that aim at maximizing a metric of the system performance (often the output power or on-axis intensity) without the knowledge of phase errors. A widely used algorithm, first applied in the context of adaptive optics for astronomy, is stochastic parallel gradient descent (SPGD) (Vorontsov and Sivokon 1998). This algorithm works by applying small random phase perturbations to the current state of the system, and examining the effect of the perturbation on the performance metric. From this observation, a control signal is generated that increases the metric, and the algorithm converges to a zero gradient point where the metric is maximized. Unlike deterministic phase control systems, the convergence time of SPGD feedback increases linearly with the number of channels, or the equivalent servo bandwidth decreases like $1 / \mathrm{N}$. Convergence time can be kept low enough by increasing the iteration rate, requiring larger computing power and actuator/detector bandwidth and lower latency. This drawback balances the main advantage of SPGD, which is the very simple detection scheme.

\subsection{Combining efficiency}

To quantify the performance of a specific coherent combining system, a single number denoted as combining efficiency is often used. Depending on the precise situation, this number can encompass different things. The most usual definition is in terms of average power in a filled aperture system, where the combined field at the output is simply the coherent sum of electric fields $E_{n}$ emitted by each path of the interferometer system. Assuming N combined beams and an ideal combining system designed for equal power in each arm, the combined average power $\mathrm{P}_{\text {comb }}$ can be written as

$$
P_{\text {comb }}=\frac{1}{N}\left|\sum_{n} E_{n}\right|^{2} \text {. }
$$

Global differences in intensities and phase mismatches among electric fields to be combined decrease the combining 
efficiency. These differences can also depend on the space variables $\mathrm{x}$ and $\mathrm{y}$ (beam profile and spatial wavefront mismatch), and on time or frequency variables $t$ and $\omega$ (spectral intensity or spectral phase mismatch, or equivalently temporal intensity and temporal phase mismatch). The combining efficiency is defined as the ratio of this combined power to the total average power emitted by the system:

$$
\eta=\frac{P_{\text {comb }}}{\sum_{n}\left|E_{n}\right|^{2}} .
$$

The impact of various intensity and phase defaults on combining efficiency has been studied both in the space (Goodno et al 2010) and time (Klenke et al 2011, Daniault et al 2012a) domains. As expected from basic interferometry, the combining efficiency is much more sensitive to phase variations than it is to intensity discrepancies among pulses to be combined. In experimental demonstrations, reported combining efficiencies are usually in the range 80\%-95\%, depending on the number of combined channels, and nonlinearity level at which fiber amplifiers are operated.

The nonlinearity level of a fiber amplifier is conveniently described by the B-integral defined as:

$$
B=\int_{0}^{L} \gamma P_{\text {peak }}(z) d z
$$

where $\mathrm{z}$ is the longitudinal coordinate, $\mathrm{L}$ is the fiber length, $\mathrm{P}_{\text {peak }}$ is the peak power in the fiber, and $\gamma$ is the nonlinear coefficient given by $\gamma=\frac{n_{2} \omega_{0}}{c A_{\text {eff }}}$. In this expression, $\mathrm{n}_{2}$ is the nonlinear index of the medium, $\omega_{0}$ is the optical angular frequency, $c$ is the speed of light in vacuum, and $A_{\text {eff }}$ is the effective area of the fiber. The B-integral corresponds to the maximum nonlinear phase shift accumulated through SPM inside the fiber. An amplifier is often considered as operating in nonlinear regime if $\mathrm{B}>\pi$. Most high-energy femtosecond fiber amplifiers operate above this limit, with a B-integral that can reach 10 rad.

The most significant effect of SPM in coherent combining systems is that it translates fluctuations of power into fluctuations of phase, which in turn hinder the combining process. Quantitative analyses of the impact of nonlinearity on the combining efficiency have been carried out in the frame of CPA systems both with analytical approaches (Klenke et al 2011) and Monte-Carlo simulations (Hanna et al 2013). These analyses also include the impact of differential group delay and group velocity dispersion on the performance metric. When coherent combining is used to scale nonlinear compression setups, the nominal B-integral level can be higher (10-20 rad), and input pulses are unchirped, leading to much more pronounced spectral profile changes. A numerical analysis of the impact of nonlinearity on the combining efficiency in this case is reported in (Guichard et al 2014).

Thermo-optic effects arising at high average power also make efficient coherent combining more difficult, because they usually lead to optical aberrations (spatial amplitude and/or phase distortions) that must be matched on the beams to be combined. For pulsed systems that primarily aim at scaling the output peak power, a variation of the definition given by equation (2) can be used, where peak powers are used to define the efficiency (Hanna et al 2013).

Modifications can be made to the simple definition of combining efficiency to take into account the added complexity of some combining geometries. For instance, in tiled aperture geometry, relative phases have an impact on the output beam shape, and the filling factor leads to a beam that exhibits side lobes, as shown in figure 4. In this case, it is more difficult to define what constitutes the useable output beam, and reported accounts of such experiments tend to use the power contained in the central lobe as a measure of combined power. Similarly, in temporal pulse combining architectures, imperfect phase matching between interferometer paths can lead to satellite pulses in the time domain on the recombined pulse, at delay values that correspond to the dividing and recombining subsystems. In this case, the useable part of the output power must be restricted to the main temporal pulse, and a temporal combining efficiency is considered in addition to the beam combining efficiency.

\section{Architectures and experimental implementations}

We now focus on the description of the variety of architectures that have been demonstrated in the past few years to perform coherent combining of femtosecond fiber amplifiers. Table 1 provides a non-exhaustive overview of references corresponding to experimental demonstrations detailed in this section. It also includes references corresponding to nonlinear compression setups detailed in section 4.1.

\subsection{Spatial: coherent beam combining}

First experimental demonstrations of coherent combining with femtosecond fiber amplifiers have used the active beam combining geometry depicted in figure 5. In these experiments, pulses emitted by a single femtosecond oscillator are stretched, and the initial beam is separated in $\mathrm{N}$ beams that are amplified in parallel in $\mathrm{N}$ amplifiers. Since these amplifiers introduce phase noise, an active detection/feedback/actuator system controls and stabilizes the phase relationship between arms of the interferometer, using either frequency tagging, or the Hänsch-Couillaud detection. Phase feedback is achieved using piezo-mounted mirrors or electro-optics phase modulators. Early demonstrations used two arms in a MachZehnder geometry (Seise et al 2010, Daniault et al 2011a, 2011b), and this number was later extended to four arms (Siiman et al 2012). By using large stretching ratios of several nanoseconds, very large mode area rodtype fibers, together with a four beam combining system, impressive performance were demonstrated, with sources delivering both large average and peak powers: $500 \mathrm{~W}, 1.8 \mathrm{GW}$ (Klenke et al 2013), $230 \mathrm{~W}, 22 \mathrm{GW}$ (Klenke et al 2014a, 2014b). Active coherent beam combining allows scaling the global performances of a single amplifier (average and peak power) by a factor $\mathrm{N}$, assuming perfect combining efficiency. 
Table 1. Overview of bibliographic references describing various architectures to perform coherent combining of femtosecond fiber amplifiers and nonlinear compression setups.

\begin{tabular}{|c|c|c|c|}
\hline & & Fiber amplifiers & Nonlinear compression \\
\hline Spatial & $\begin{array}{l}\text { Active } \\
\text { Passive }\end{array}$ & $\begin{array}{l}\text { (Seise } \text { et al 2010) } \\
\text { (Daniault } \text { et al 2011a) } \\
\text { (Siiman } \text { et al 2012) } \\
\text { (Klenke } \text { et al 2014a) } \\
\text { (Ramirez } \text { et al 2015) } \\
\text { (Daniault } \text { et al 2011b) } \\
\text { (Zaouter } \text { et al 2012) }\end{array}$ & (Klenke et al 2014b) \\
\hline Temporal & $\begin{array}{l}\text { Active } \\
\text { Passive }\end{array}$ & $\begin{array}{l}\text { (Kienel et al 2014a) } \\
\text { (Zaouter } \text { et al 2013) }\end{array}$ & $\begin{array}{l}\text { (Klenke et al 2013) } \\
\text { (Jacqmin } \text { et al 2015) }\end{array}$ \\
\hline Spatial + Temporal & $\begin{array}{l}\text { Active } \\
\text { Passive }\end{array}$ & $\begin{array}{l}\text { (Kienel } \text { et al 2015) } \\
\text { (Daniault et al 2012b) } \\
\text { (Guichard } \text { et al 2015) }\end{array}$ & (Guichard et al 2013b) \\
\hline Spectral synthesis & $\begin{array}{l}\text { Active } \\
\text { Passive }\end{array}$ & $\begin{array}{l}\text { (Chang et al 2013) } \\
\text { (Guichard et al 2013a) } \\
\text { (Rigaud } \text { et al 2013) }\end{array}$ & (Krausst et al 2010) \\
\hline
\end{tabular}

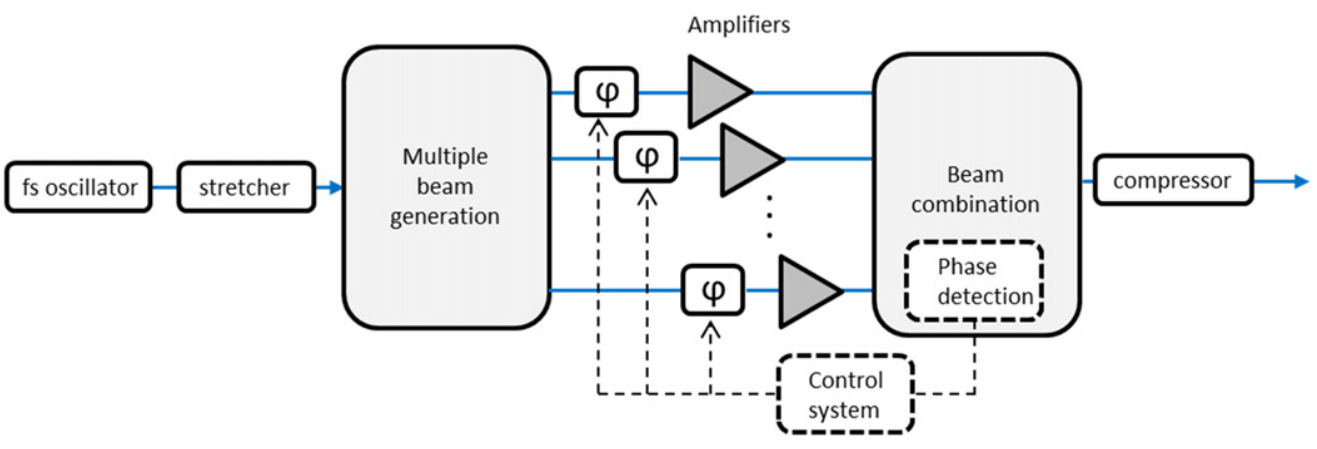

Figure 5. Active coherent beam combining system.

A key factor in these experiments is the ability to replicate accurately each arm and design the system so that upon recombination, the temporal/spectral and spatial properties of the amplified beams are as identical as possible. The guiding nature of the gain medium is particularly helpful in this sense, as it guarantees the beam quality, largely independent on operating power. In the time domain, some care is required to balance the amount of group-velocity dispersion introduced by each arm, and a tunable delay line is usually included in each arm to fine-tune the group delay. In highly nonlinear systems $(\mathrm{B}>\pi \mathrm{rad})$, the amount of accumulated nonlinear phase should also be matched to maximize combining efficiency (Daniault et al 2012a, 2012b). It is worthwhile to point out that the polarization state at the output of amplifiers must also be fixed, which can be guaranteed by the use of polarization-maintaining fibers. The combining efficiency in these experiments, that all use filled aperture combining using two port optical components, depends on the number of arms combined, the level of nonlinearity and the average power, but generally lies in the $80 \%-95 \%$ range. The bandwidth of the electronic feedback systems in these demonstrations is of the order of $1 \mathrm{kHz}$, sufficient to effectively suppress acoustic and thermal noise in a laboratory environment.

A recent proof-of-principle experiment (Ramirez et al 2015) using the same active beam combining architecture differs from previous works in several points. It integrates the fiber amplifiers in a single structure by using a multicore fiber that can be cladding-pumped by a single high power diode laser system. The main advantage is that relative phase fluctuations between beams to be combined are much reduced since the cores are very close to one another. This has been experimentally characterized, showing that it is sufficient to correct long term phase drifts with a bandwidth of $2 \mathrm{~Hz}$ to phase lock the system. The second difference is that combining is performed using the tiled aperture geometry using a microlens array that collimates the seven output beams from the multicore fiber. The tiling geometry, and in particular the filling factor, limits the theoretical combining efficiency to $76 \%$ in this case, and the experimentally obtained efficiency is $50 \%$. The difficulty in controlling the relative group delay in long and twisted multicore fibers calls for the use of straight and short rodtype multicore fibers such 


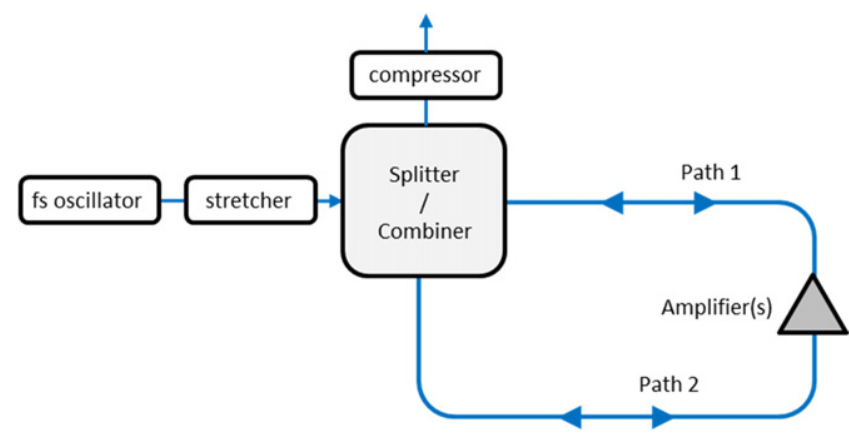

Figure 6. Sagnac amplifier configuration for passive beam combining.

as those used in (Otto et al 2014), that could be a promising way to efficiently integrate femtosecond fiber coherent beam combining systems.

As an alternative to active coherent beam combining, passive architectures, implying that phase stabilization is an inherent characteristic of the system, have been proposed and demonstrated in the context of fiber femtosecond amplifiers. The combination of two amplified beams is accomplished without the need for an active detection/feedback mechanism. The principle of operation is to use an amplifying interferometer in which all arms are composed of the same overall optical path, the only difference between arms being the order in which each path element is encountered. This guarantees that, upon combination, spectral phases are matched to all orders. The most obvious example of such an interferometer, and the only one that has been experimentally implemented to date, is the Sagnac interferometer, shown in figure 6 . This idea was proposed 20 years ago in the context of excimer amplifiers in the $\mathrm{CW}$ regime (Szatmari and Simon 1993). In this geometry, the splitter/combiner launches the two beams in a counterpropagating fashion in the Sagnac loop that includes one or several fiber amplifiers. The symmetry of both paths is an important point to guarantee a high value of the combining efficiency (Daniault et al 2011b). These setups are intrinsically immune to phase noise frequency components below the inverse roundtrip time in the interferometer, corresponding to tens of $\mathrm{MHz}$ in meter-sized setups. This property has ensured robust phase locking of femtosecond beams in different contexts, e.g. allowing the generation of $600 \mu \mathrm{J}, 300 \mathrm{fs}$ pulses at $60 \mathrm{~W}$ average power level in a compact setup (Zaouter et al 2012).

As detailed in (Hanna et al 2013), it is possible to devise interferometer arrangements to extend this principle to combine more beams and amplifiers. However, this is of limited interest in the case of fiber amplifiers, since each beam must propagate through each amplifier, accumulating nonlinearity, which is precisely the phenomenon that limits the achievable performances. However, this could be an interesting perspective in other systems such as bulk media-based multipass amplifiers, especially when limited by low gain and energy extraction capacity, as first demonstrated in reference (Papadopoulos et al 2015).

\subsection{Temporal: divided pulse amplification}

Divided pulse amplification (DPA) consists in creating several replicas of an incoming pulse in the time domain, amplify the sequence of pulses, and recombine them coherently, as depicted in figure 7 . Note that in this configuration, a single beam is used throughout the system. DPA can therefore not be considered as a way to scale the average power of a given system. This process reduces the peak power by a factor equal to the number of replicas. Since it consists in distributing the power over time, it can be also seen as a discrete version of CPA. DPA has first been proposed in the context of picosecond fiber amplifiers (Zhou et al 2007), where CPA is difficult to use because of the narrow optical bandwidth available.

One implementation of DPA uses the same optical subsystem to perform both pulse division and combining, as shown in figure 7 . This allows automatic balance of the time interferometer, with pulses experiencing complementary delays on the way forward and back into the pulse division system. This feature bears resemblance with the Sagnac passive beam combining system described above. Passive DPA has been implemented in a variety of contexts, including picosecond fiber amplifiers (Kong et al 2012), stretcher free 'parabolic' fiber femtosecond amplifiers (Daniault et al 2012b), and femtosecond fiber CPAs (Zaouter et al 2013). In the two first examples, birefringent crystals were used to divide and recombine the pulses, because the pulse duration was below a few picoseconds. The largest demonstrated number of replicas generated with crystals is 32 . In the case of CPA, freespace delay lines were implemented to accommodate the stretched pulse duration of several hundred picoseconds. An experiment using passive DPA and CPA has allowed the generation of $430 \mu \mathrm{J}$ pulses from a rodtype fiber amplifier (Zaouter et al 2013). In all cases DPA has allowed peak power scaling by reducing the level of optical nonlinearity and/or increasing the damage threshold in terms of pulse energy.

Limitations of the DPA architecture are related with the fact that successive pulses in the train might not experience the exact same condition upon propagation in the amplifier. For example, at high nonlinearity level ( $\mathrm{B}>\pi \mathrm{rad}$ ), small deviations in intensity of the generated replicas can translate into phase shifts through SPM, hindering the temporal combining process, and the systems becomes more sensitive to any imperfection (Guichard et al 2014). Another unavoidable limiting effect is gain saturation in the fiber amplifying medium (Kienel et al 2013). When the pulse energy becomes of the order of the amplifier saturation energy, the gain experienced by successive replicas decreases, leading to differences in intensity that can also be turned into phase differences through SPM or Kramers-Krönig induced index change. The train of amplified pulses that propagates through the combiner is therefore non-uniform both in intensity and phase, and combining efficiency decreases. In passive systems, this saturation effect can be compensated in the case of only two temporal replicas by adjusting the intensity level between replicas at the input. For a larger number of replicas, the compensation is only partial: there are not enough 


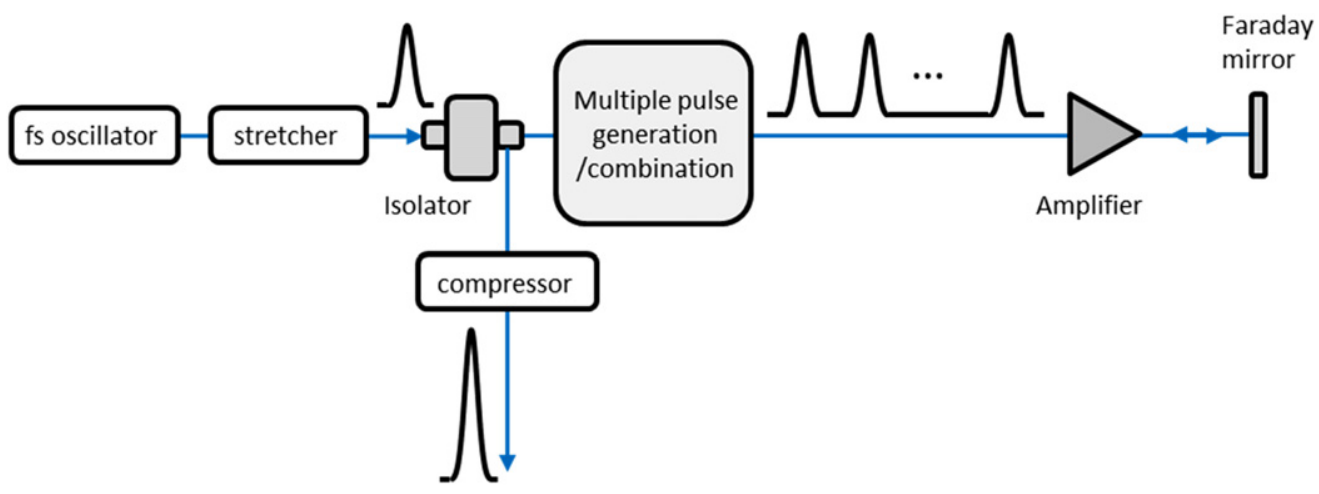

Figure 7. Passive divided-pulse amplification.

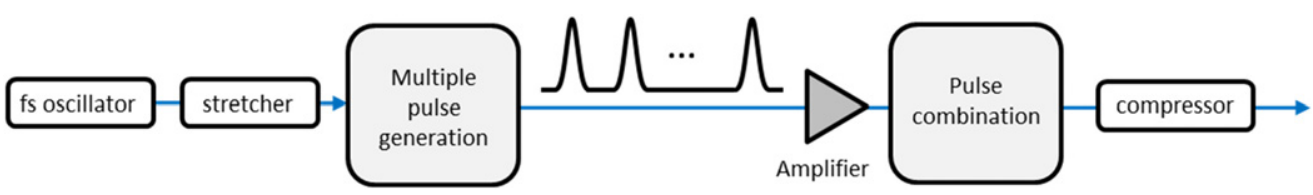

Figure 8. In-line divided-pulse amplification.

experimental degrees of freedom to adjust the amplitude of each replica, since the same subsystem is used to split and combine, and the number of replicas increases exponentially with the number of delay lines used. Implementations of passive DPA setups including optical components (such as a Pockels cell) allowing intensity control of each pulse can be imagined, but have not been demonstrated to our knowledge.

To circumvent this problem, another implementation of DPA using separate divider and combiner subsystems was proposed, as shown in figure 8 . If the splitter and combiner are based on bulk birefringent crystals, phase differences between the ordinary and extraordinary polarization states induced by such crystals in a well-controlled environment are stable enough to implement the system without active phase control among pulse replicas. However, to use this scheme in conjunction with CPA, freespace delay lines are used both to divide and combine, making the system equivalent to a freespace Mach-Zehnder with meter-sized arm lengths. In this case, active phase control using techniques such as frequency tagging is required. Kienel et al 2014a reports on the generation of $1.25 \mathrm{~mJ}$ femtosecond pulses using four replicas, a stretched pulse duration of $2 \mathrm{~ns}$, and a rodtype fiber with a mode-field diameter of $75 \mu \mathrm{m}$. Although active DPA increases the number of useable replicas even in the case of strong gain saturation, the number of degrees of freedom on the intensity of the input pulse train must be equal to $\mathrm{N}-1$, where $\mathrm{N}$ is the total number of replicas. Pulse train shaping methods allowing the control of the intensity of each pulse are needed to scale this idea to a large number of replicas.

\subsection{Spatial and temporal/active and passive}

Looking at both previous sections, it has been a tempting idea to use simultaneously spatial and temporal approaches in order to further scale the number of replicas that can be amplified and combined. This leads to 'spatio-temporal' or 'multi-dimensional' coherent combining approaches. Since DPA dividing and combining subsystems generate a single output beam, reported demonstrations first implement temporal replicas generation before splitting each temporal replica in several beams. After amplification, beams are first combined in space, and then temporal replicas are re-synchronized to generate a single output pulse. In these experiments, special attention must be paid to take into account the fact that the polarization state somehow encodes the temporal replica. In standard DPA systems, the amplifying medium must not exhibit polarization-dependent properties such as birefringence (polarization-maintaining properties) or polarization-dependent gain or loss. Indeed, these properties introduce differences in behaviors among replicas that prevent proper combination. An advantage of implementing both DPA and beam combining is that it makes the architectures compatible with polarization maintaining fiber amplifier or fibers exhibiting polarization-dependent gain and loss: temporal replicas have orthogonal polarization states that can be separated in two beams by a polarizer oriented at $45^{\circ}$, thereby generating two beams with a single linear polarization state that can be sent into PM fiber amplifiers. Spatio-temporal combining has been demonstrated both in fully passive and fully active architectures.

The passive approach consists in combining a Sagnac interferometer with a passive DPA setup. Since both approaches are limited in current practical setups to two replicas, it is a simple way to conceive an entirely passive coherent combining system that is compatible with highenergy fiber femtosecond amplifiers and uses 4 replicas. A recent demonstration (Guichard et al 2015) implements this idea in a CPA system, using freespace delay lines to generate the temporal replicas, with a Sagnac interferometer including two $0.8 \mathrm{~m}$-long rodtype fiber amplifiers. The setup allows the generation of $1.1 \mathrm{~mJ} 300 \mathrm{fs}$ pulses with a combining efficiency of $90 \%$, illustrating the relevance of the passive 
architecture: the energy is scaled by a factor of four compared to performances achieved without any combining strategies, without the need for any active opto-electronic feedback loop system.

The active approach, on the other hand, consists in implementing an active DPA setup together with active coherent beam combining. A recent experiment (Kienel et al 2015) demonstrates the concept with four temporal replicas and 2 amplifying arms forming a Mach-Zehnder interferometer, therefore using 8 overall replicas. Three active phase control systems are necessary to perform coherent combining, 2 for the temporal and 1 for the spatial multiplexing dimensions. The active phase control system in this case uses the LOCSET method. Achieved combining efficiencies are between $75 \%$ and $95 \%$, depending on the operation regime: $75 \%$ efficiency is obtained only for extreme parameters in terms of B-integral and/or energy compared to the saturation energy of the amplifiers. The added complexity of the setup compared to the passive approach is balanced by straightforward scaling capabilities of the technique in the spatial dimension.

Finally, the potential of combination of the active and passive techniques in the same setup seems to be an appealing perspective. In fact, the simplicity and the inherent robustness of passive combination setups could allow their use as the unit element integrated in an active combination configuration where two or more of these passively combined systems could be actively coherently combined, further scaling the so far obtained performance based on each approach separately.

\subsection{Spectral: pulse synthesis}

This section is devoted to the description of experiments that perform coherent addition of ultrafast optical fields that have different optical spectra. It deviates from previous sections in the sense that the combined pulse is not a scaled version of supposedly identical lower energy pulse replicas, since its spectral content is broader than that of individual pulses. However, this concept, sometimes denoted as spectral coherent combining or pulse synthesis, still requires the control of relative phases and group delays between beams to be combined. It can be seen as the temporal/spectral equivalent to tiled aperture combining in the spatial/wavevector domains: tiling is done in the spectral domain, resulting in the synthesis of a shorter pulse in the time domain, just as tiling in the near field results in the synthesis of a single narrower lobe in the far field. Most of the experiments performed in this area follow a schematic exactly identical to active coherent beam combining depicted in figure 4 , except that the splitter and combiner elements are spectrally selective, and route different parts of the initial broad spectrum into different arms of the amplifying interferometer. This can be done, for example, with dichroic mirrors. One exception to this design is a pioneer experiment (Shelton et al 2001) in which two Ti:Sa oscillators emitting different spectra were combined, necessitating stabilization and synchronization of both the repetition rate and the carrier-envelope frequency.
This experiment demonstrated the shortening of the combined pulse compared to the initial ones.

Since then, a number of experiments using a single ultrafast oscillator feeding several optical parametric amplifiers optimized at well separated wavelengths through supercontinuum generation (Huang et al 2011), or several fiber-based nonlinear stages (Krausst et al 2010), have allowed the generation of optical transients with extreme durations comparable or even shorter than the optical cycle at the centroid wavelength. The characterization of such optical transients, and control of the spectral content, in particular the spectral phase, remains a very challenging task (Chia et al 2014). The goal of these large scale experiments is to generate extremely short pulses with enough energy to perform various high field experiments, and power scaling aspects are usually not considered.

More recently, few pulse synthesis experiments were carried out in the frame of ultrafast ytterbium-doped fiber amplifiers. The purpose of the architecture shifts to simultaneously scale the power of the system and compensate gain narrowing by isolating spectral slices of the seed pulse and send them in several fiber amplifiers. Indeed, the effect of gain narrowing on narrower spectra tends to be reduced at a given gain value. This technique can be viewed as an alternative to spectral filtering methods that can be used to precompensate the spectral dependence of gain in an amplifier. The pros and cons of spectral synthesis vs spectral shaping is a complex matter that involves parameters of the experiment such as total amount of gain, homogeneous and inhomogeneous behavior of the gain medium, and dynamic range of the spectral shaper component. These parameters are important in determining the achievable output bandwidth, along with amplified spontaneous emission and parasitic laser oscillation characteristics of the amplification system.

One of the specifics of spectral coherent combining is that, depending on the amount of spectral overlap among the pulses to be combined, the output pulse energy, or equivalently the output average power, can exhibit very little dependence on the relative phase. In the extreme case of nonoverlapping spectra, only the peak power of the output pulse depends on the phase, and phase sensing techniques must be adapted to this situation. A first experimental proof-of-principle (Chang et al 2013) was carried out with 3 amplifying arms actively combined and all-fiber components. The LOCSET phase locking technique was used with either a linear photodiode or a two-photon absorption photodiode to detect variations of the combined pulse energy or peak power, successfully phase locking non-overlapping spectra.

Another experiment (Guichard et al 2013a) combining two portions of spectrum separately amplified, as shown in figure 9, was carried out and demonstrated that spectral combining allows the generation of shorter pulses than a single amplifier. Partially overlapping spectra allowed the use of the LOCSET technique with linear photodiode detection. However, to increase the signal to noise ratio of phase detection, a grating was used before the photodiode to isolate the overlapping spectral part. The generation of amplified 

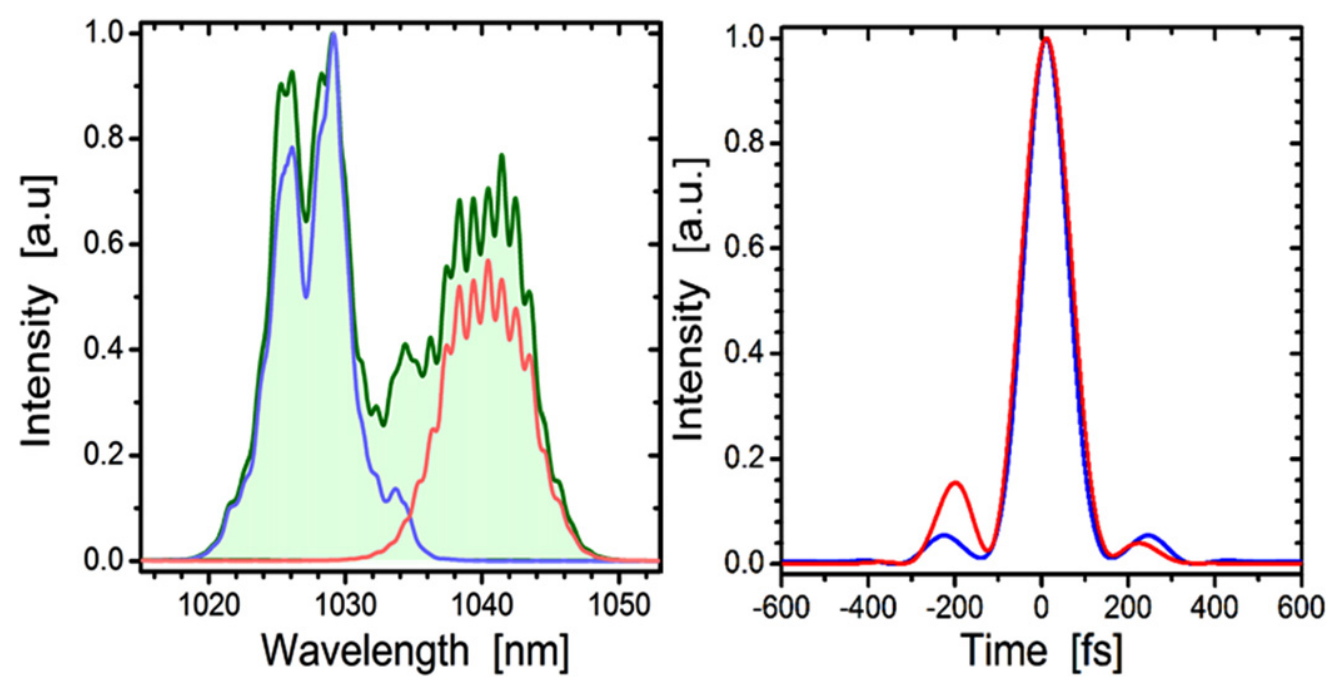

Figure 9. Left: spectra of each amplifier (red and blue) and combined spectrum (green). Right: synthesized temporal pulse profile (red) Fourier-transform limited profile (blue). From (Guichard et al 2013a).

$130 \mathrm{fs}$ pulses at a gain value of $30 \mathrm{~dB}$ was achieved, showing the potential of the technique to overcome gain narrowing.

A third experiment implemented spectral pulse synthesis in an in-line multicore Yb-doped fiber (Rigaud et al 2013). Because of the large number of spectral channels combined in this experiment (12), dichroic mirrors are advantageously replaced by a grating-based subsystem to perform spectral division and combining, at the expense of residual angular chirp at the output. The system is core-pumped by 12 diffraction-limited pump diodes, and reduced phase fluctuations in the multicore fiber allows low bandwidth relative phase adjustment using a deformable mirror.

It should be highlighted that spectral coherent combining, when used in conjunction with chirped pulse amplification, does not allow energy scaling of a peak power-limited system: when a highly stretched pulse is split in spectral slices, the time-to-spectrum mapping introduced by group-velocity dispersion implies that the pulse is also sliced in the temporal domain, with no reduction of the peak power fed to each arm. However, pulse synthesis architectures still allow average power scaling, and an increase of the peak power due to larger output optical bandwidth.

\section{Coherent addition of ultrafast pulses: other aspects}

We now describe laser sources that use concepts of coherent addition of ultrafast pulses, but are not strictly related to fiber amplifiers. The first section describes the use of coherent combining to overcome energy limitations in temporal nonlinear compression setups. The second section deals with the use of passive resonant cavities that, from an incident modelocked laser pulse train, coherently build up a recirculating intracavity optical beam carrying a large average power/pulse energy.

\subsection{Coherent combining in nonlinear temporal compression systems}

Temporal nonlinear compression is a well-established way to reduce the pulse duration at the output of femtosecond laser sources. The principle is to propagate femtosecond pulses in a nonlinear Kerr medium that broadens the spectrum through SPM. Subsequent suppression of the induced chirp with appropriate dispersive elements then leads to pulse shortening. To avoid detrimental effects on the beam quality imparted by the spatial Kerr effect, SPM is usually induced in an optical waveguide that also maintains a high optical intensity over long interaction lengths. Various waveguide types have been used including solid-core fibers, and gasfilled hollow-core fibers and capillaries. Compressible pulse energy is limited to the $\mu \mathrm{J}$ level in solid-core fibers by effects such as optical damage and self-focusing, and to the $\mathrm{mJ}$ level in hollow-core waveguide due to the onset of gas ionization. It is therefore a natural idea to apply the concepts developed in the context of energy-limited fiber amplifiers to post compression setups. In fact, almost all types of architectures described in the previous part have been applied to compression setups in the last few years. The most striking difference is the higher level of nonlinearity and the larger optical bandwidth over which phase matching must be maintained to guarantee a large value of combining efficiency.

Active coherent beam combining has been recently demonstrated in the context of nonlinear temporal compression (Klenke et al 2014a, 2014b). The experimental layout is exactly the one depicted in figure 5 with two arms forming a Mach-Zehnder, the fiber amplifiers being replaced by passive large mode area silica fibers where SPM is experienced by the pulses to be compressed. Obtained combining efficiencies are in the range $75 \%-80 \%$, while initial $300 \mathrm{fs}$ pulses are compressed down to $30 \mathrm{fs}$, at an energy level of $1.1 \mu \mathrm{J}$. This level is above the self-focusing limit of a single fiber. This experiment shows the relevance of coherent beam combining 

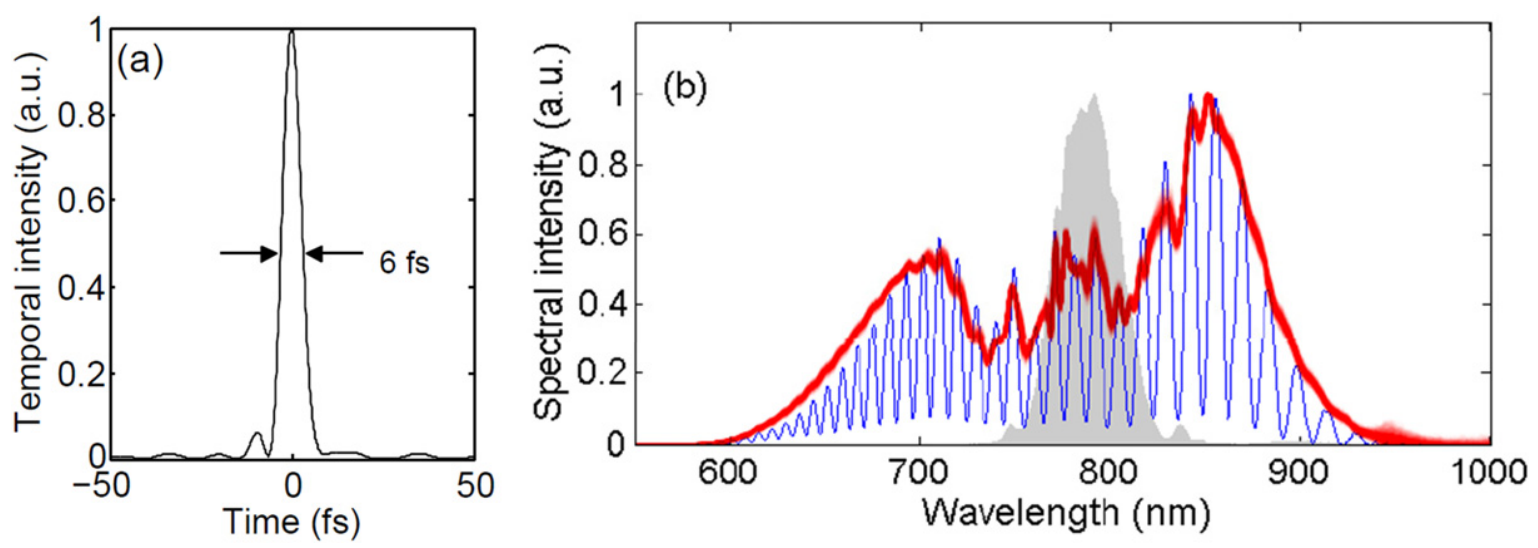

Figure 10. Left-Temporal profile of the compressed pulse. Right-Spectrum of the compressed pulse (red) Spectrum before combination showing delay fringes (blue), and input spectrum (grey). Adapted From (Jacqmin et al 2015).

even for pulses exhibiting spectral bandwidth $>50 \mathrm{~nm}$ at a central wavelength of $1030 \mathrm{~nm}$.

The divided pulse technique has also been applied to nonlinear compression setups. Reference (Klenke et al 2013) reports the use of a passive DPA setup similar to the layout shown in figure 7 that generates four replicas with freespace delay lines to compress $320 \mathrm{fs}$ down to $95 \mathrm{fs}$ in a large mode area solid-core fiber, with an efficiency estimated to be around $75 \%$. This non optimal value is attributed by the authors to issues with alignment of the freespace delay lines that translate into difference in coupling among replicas to the nonlinear fiber. Nevertheless, the final obtained peak power is $10 \mathrm{MW}$, corresponding to $1 \mu \mathrm{J}$, again beyond self-focusing and damage thresholds in the optical fiber.

Going further in number of replicas, reference (Guichard et al 2013b) demonstrates the use of a spatio-temporal coherent combining setup using passive DPA and a Sagnac interferometer, following the architecture described in section 3.3. The nonlinear medium used is again an undoped large mode area rodtype fiber. In this experiment, pulse division is achieved with thick (up to $20 \mathrm{~mm}$ ) birefringent crystals, since the input pulse duration is below 1 ps. This avoids alignment issues and allows generation of 16 temporal replicas in a reasonably simple setup. The Sagnac arrangement doubles the number of replicas to reach 32 . This high number of replicas allows temporal compression to generate $71 \mathrm{fs} 7.5 \mu \mathrm{J}$ pulses, corresponding to $86 \mathrm{MW}$ peak power. As already observed in fiber amplifier context, alignment tolerances generally decrease as the level of nonlinearity increases. Reference (Guichard et al 2014) reports on a detailed analysis of the limitations of these setups due to two phenomena: nonequal intensity distribution among the train of pulse replicas, and differential dispersion. This last effect is specific to divided-pulse scheme using birefringent crystals, and is related to the fact that dividing and combining paths exhibit slightly different dispersion properties. In the presence of nonlinearity, this breaks the symmetry between the division/ combination processes and can limit the efficiency.

Finally, a recent experiment (Jacqmin et al 2015) describes the use of the passive divided-pulse architecture in

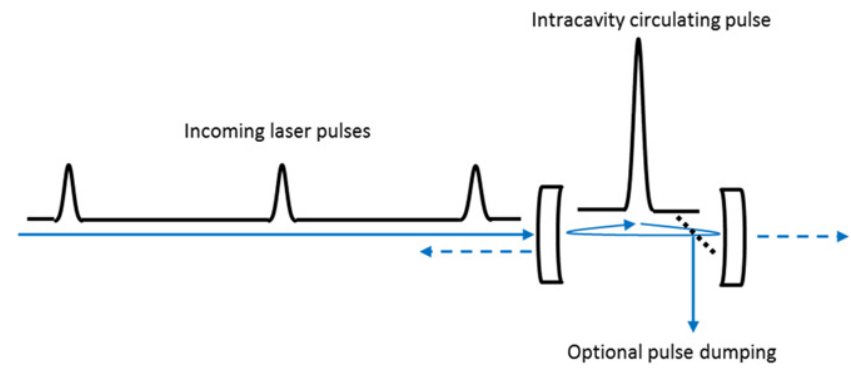

Figure 11. Schematic of a passive enhancement cavity.

the context of Ti:Sa-generated pulses compressed down to few-cycle duration in hollow-core capillaries, following a theoretical study (Wang et al 2014). Temporal division and combination of the input $30 \mathrm{fs}$ pulses is performed using separate thin $(500 \mu \mathrm{m})$ calcite crystals, so that differential dispersion is negligible even for few cycle pulses. The experiment demonstrates that it is possible to use coherent combining ideas with 6 fs pulses, as shown in figure 10, with efficiencies greater than $90 \%$, at a final energy per pulse of $0.6 \mathrm{~mJ}$. Moreover, carrier-envelope phase (CEP) measurements indicate that the divided-pulse compressor does not introduce any CEP drifts or fluctuations, an essential feature for few-cycle pulse sources.

\subsection{Cavity enhancement/pulse stacking}

Passive resonant optical cavities can be used to accumulate and concentrate the power emitted by a laser inside the cavity volume. This is possible because of the phase-coherent properties of optical pulse trains emitted by a mode-locked laser: since there is a fixed phase relationship between successive pulses in the train, pulses can interfere constructively with each other to build-up the recirculating intracavity field. In this sense it constitutes a coherent combining concept in the time domain, where division is performed by the femtosecond laser oscillator cavity, and combining is achieved by the resonant passive cavity. A schematic of the principle of cavity enhancement is shown in figure 11. 
Enhancement cavities have been used in the $\mathrm{CW}$ regime for a long time, but have been applied to ultrafast lasers only during last decade: in single-frequency regime, enhancement is obtained if the input optical frequency matches one of the resonances of the cavity. In the femtosecond regime, the frequency comb corresponding to the input excitation spectrum must coincide with the cavity-defined frequency comb. This condition is much harder to achieve, and, depending on the cavity finesse and optical bandwidth, requires active stabilization of both the CEP frequency and the repetition rate of the laser and/or the cavity. Moreover, the optical bandwidth over which the frequency combs of the feeding laser and the cavity overlap is essentially determined by dispersion in the cavity, which results in a non-uniform cavity frequency comb. This effect reduces the intracavity power enhancement that can be expected, and increases the pulsewidth of the circulating pulse. Other critical design parameters for enhancement cavities include cavity losses, input coupler, intracavity optical nonlinearities, thermo-optic properties at high power, and cavity geometry in terms of stability and beam sizes. Two types of experiments have been reported that make use of the cavity enhancement idea.

The first type of experiments consists in locating the final interaction, where high intensity pulses are needed and used, inside the enhancement cavity. If this interaction has low efficiency, it contributes little to the losses of the cavity and still allows for efficient pulse buildup. Another condition to use this architecture is that the interaction should leave the optical beam intact, so that spatial mode matching between the cavity and the input beam is still possible. Applications where this geometry has been successfully used include highharmonic generation (Jones et al 2005, Pupeza et al 2013) and Compton or Thomson scattering (Couprie et al 1999, Pogorelsky et al 2014). In this context, cavity enhancement factors of several hundred to several thousands were demonstrated in temporal regimes ranging from sub-100 fs pulses to ps pulses (Pupeza et al 2010, Börzsönyi et al 2013), allowing the optical peak power in the cavity to be scaled to hundreds of MW directly at the oscillator repetition rate of several tens of MHz. In several experiments, optical intensities of several $10^{14} \mathrm{~W} \mathrm{~cm}^{-2}$ at the intracavity focus were obtained. In terms of average intracavity power, the performances reached so far lie in the $1 \mathrm{~kW}-1 \mathrm{MW}$ range, with $640 \mathrm{~kW}$ average power demonstrated recently (Carstens et al 2014). Further power scaling is limited by thermo-optic effects at the cavity mirrors, optical nonlinearity and damage, and dispersion control.

The second use of passive cavities consists in enhancing the input optical pulses by stacking them onto each other in the cavity for a certain number of roundtrip times, then ejecting the recirculating pulse out of the cavity using a cavity dumper (Potma et al 2003). The output beam has a reduced repetition rate, but features higher pulse energy, and the cavity has acted as an energy concentrator in time. This feature is ideally suited to fiber-based sources that can easily handle large average powers but are limited in terms of pulse energy. The overall power efficiency of this conversion depends, among other parameters, on the repetition rate down-counting and intracavity losses.

Finally, a recent proposition consists in using one or several Gires-Tournois interferometer cavities to concentrate the energy of an appropriately amplitude and phase-shaped mode-locked train of pulses in a single output stacked pulse (Zhou et al 2015). The main advantage of this proposal is that the stacked pulse is passively coupled out from the resonant optical system. On the downside, the largest theoretical power enhancement that can be obtained from a single interferometer is 2.62. Using multiple cavities allows larger power enhancement factors, at the price of a setup complexity that grows considerably.

\section{Conclusion}

Coherent combining ideas applied to ultrafast laser sources appear as one of the most promising path to next generation femtosecond laser sources delivering both high average and high peak power. Large-scale projects aiming at exploiting coherent combining to build new laser facilities delivering joule-class pulses at repetition rates of several tens of $\mathrm{kHz}$ are being pursued (Brocklesby et al 2014). The most obvious way towards this ambitious goal is to use active spatial coherent combining and simply scale the number of fiber amplifiers to a large number from 100 to 10000 depending on specifics of the system design such as fiber mode field diameter, stretching ratio, use of DPA, or use of multicore fibers. This calls for collective approaches for all aspects of the combining system, notably for phase sensing, phase control, and beam combining geometry. Another proposed system design (Breitkopf 2014) consists in using a passive enhancement cavity with active dumping, to convert $\mathrm{mJ}$ pulses at $10 \mathrm{MHz}$ obtained by active coherent combining of a smaller number of fiber amplifiers (about twenty) into J pulses at $10 \mathrm{kHz}$. The critical technological point is to perform cavity dumping while minimizing intracavity losses to maintain a reasonable power efficiency of the repetition rate down converter.

Although first experimental demonstrations have been done with Yb-doped fiber amplifiers systems, coherent combining of ultrafast pulses is being applied to nonlinear compression setups, Tm-doped fiber amplifiers (Gaida et al 2015), bulk amplifiers (Kienel et al 2014b, Papadopoulos et al 2015) and ultrafast oscillators (Lamb et al 2014). Concepts using coherent addition of ultrafast optical fields are borrowed from and applied outside the scope of fiber laser sources, and are rapidly becoming a general toolbox in ultrafast optics.

\section{Acknowledgments}

We acknowledge support from Investissement d'avenir Labex PALM PulseSynth (grant number ANR-10-LABX-0039), and ANR MultiFemto (grant number ANR-11-BS09-02801). 


\section{References}

Augst S J, Fan T Y and Sanchez A 2004 Coherent beam combining and phase noise measurements of ytterbium fiber amplifiers Opt. Lett. 29 474-6

Börzsönyi A et al 2013 External cavity enhancement of picosecond pulses with 28000 cavity finesse Appl. Opt. 52 8376-80

Bourderionnet J, Bellanger C, Primot J and Brignon A 2011 Collective coherent phase combining of 64 fibers Opt. Express 19 17053-8

Breitkopf S 2014 A concept for multiterawatt fibre lasers based on coherent pulse stacking in passive cavities Light: Sci. Applications 3 e 211

Brocklesby W S et al 2014 ICAN as a new laser paradigm for high energy, high average power femtosecond pulses Eur. Phys. J. Special Topics 223 1189-95

Carstens $\mathrm{H}$ et al 2014 Megawatt-scale average-power ultrashort pulses in an enhancement cavity Opt. Lett. 39 2595-8

Chang W-Z, Zhou T, Siiman L A and Galvanauskas A 2013 Femtosecond pulse spectral synthesis in coherently-spectrally combined multi-channel fiber chirped pulse amplifiers $O p t$. Express 21 3897-910

Cheung E C, Weber M and Rice R R 2008a Advanced solid-state photonics OSA Technical Digest Series (CD) (Optical Society of America) paper WA2

Cheung E C, Ho J G, Goodno G D, Rice R R, Rothenberg J, Thielen P, Weber M and Wickham M 2008b Diffractiveoptics-based beam combination of a phase-locked fiber laser array Opt. Lett. 33 354-6

Chia S-H, Cirmi G, Fang S, Rossi G M, Mücke O D and Kärtner F X 2014 Two-octave-spanning dispersion-controlled precision optics for sub-optical-cycle waveform synthesizers Optica 1 315-22

Couprie M, Nutarelli D, Roux R, Visentin B, Nahon L and Bakker R 1999 Gamma rays produced by intra-cavity inverse Compton scattering of a storage ring free-electron laser J. Phys. B: At. Mol. Opt. Phys. 32 5657-67

Daniault L, Hanna M, Lombard L, Zaouter Y, Mottay E, Goular D, Bourdon P, Druon F and Georges P 2011a Coherent beam combining of two femtosecond fiber chirped-pulse amplifiers Opt. Lett. 36 621-3

Daniault L, Hanna M, Papadopoulos D N, Zaouter Y, Mottay E, Druon F and Georges P 2011b Passive coherent beam combining of two femtosecond fiber chirped-pulse amplifiers Opt. Lett. 36 4023-5

Daniault L, Hanna M, Lombard L, Zaouter Y, Mottay E, Goular D, Bourdon P, Druon F and Georges P 2012a Impact of spectral phase mismatch on femtosecond coherent beam combining systems Opt. Lett. 37 650-2

Daniault L, Hanna M, Papadopoulos D N, Zaouter Y, Mottay E, Druon F and Georges P 2012b High peak-power stretcher-free femtosecond fiber amplifier using passive spatio-temporal coherent combining Opt. Exp. 20 21627-34

Eidam T, Rothhardt J, Stutzki F, Jansen F, Hädrich S, Carstens H, Jauregui C, Limpert J and Tünnermann A 2011 Fiber chirpedpulse amplification system emitting $3.8 \mathrm{GW}$ peak power $O p t$. Exp. 19 255-60

Fan T Y 2005 Laser beam combining for high-power, high-radiance sources IEEE J. Sel. Top. Quantum Electron. 11567

Flores A, Pulford B, Robin C, Lu C A and Shay T M 2013 Coherent beam combining of fiber amplifiers via LOCSET Coherent Laser Beam Combining ed A Brignon (Weinheim: WileyVCH) pp 277-301

Gaida C, Kienel M, Müller M, Klenke A, Gebhardt M, Stutzki F, Jauregui C, Limpert J and Tünnermann A 2015 Coherent combination of two Tm-doped fiber amplifiers Opt. Lett. 40 2301-4
Goodno G D, Shih C-C and Rothenberg J E 2010 Perturbative analysis of coherent combining efficiency with mismatched lasers Opt. Exp. 18 25403-14

Guichard F, Hanna M, Lombard L, Zaouter Y, Hönninger C, Morin F, Druon F, Mottay E and Georges P 2013a Twochannel pulse synthesis to overcome gain narrowing in femtosecond fiber amplifiers Opt. Lett. 38 5430-3

Guichard F, Zaouter Y, Hanna M, Morin F, Hönninger C, Mottay E, Druon F and Georges P 2013b Energy scaling of a nonlinear compression setup using passive coherent combining Opt. Lett. 38 4437-40

Guichard F, Hanna M, Zaouter Y, Papadopoulos D N, Druon F and Georges P 2014 Analysis of limitations in divided-pulse nonlinear compression and amplification J. Sel. Top. Quantum Electron. 207600405

Guichard F, Zaouter Y, Hanna M, Mai K-L, Morin F, Hönninger C, Mottay E and Georges P 2015 High-energy chirped- and divided-pulse Sagnac femtosecond fiber amplifier Opt. Lett. 40 89-92

Hanna M, Papadopoulos D N, Daniault L, Druon F, Georges P and Zaouter Y 2013 Coherent beam combining in the femtosecond regime Coherent Laser Beam Combining ed A Brignon (Weinheim: Wiley-VCH) pp 277-301

Hänsch T W and Couillaud B 1980 Laser frequency stabilization by polarization spectroscopy of a reflecting reference cavity $O p t$. Commun. 35 441-4

Hartl I, Marcinkevicius A, McKay H A, Dong L and Fermann M E 2009 Coherent beam combination using multi-core leakagechannel fibers Advanced Solid-State Photonics, OSA Technical Digest Series (CD) (Optical Society of America) paper TuA6

Huang S-W et al 2011 High-energy pulse synthesis with sub-cycle waveform control for strong-field physics Nat. Photonics 5 475-9

Jacqmin H, Jullien A, Mercier B, Hanna M, Druon F, Papadopoulos D and Lopez-Martens R 2015 Passive coherent combining of CEP-stable few-cycle pulses from a temporally divided hollow fiber compressor Opt. Lett. 40 709-12

Jones R J, Moll K D, Thorpe M J and Ye J 2005 Phase-coherent frequency combs in the vacuum ultraviolet via high-harmonic generation inside a femtosecond enhancement cavity Phys. Rev. Lett. 94193201

Kienel M, Klenke A, Eidam T, Baumgartl M, Jauregui C, Limpert J and Tünnermann A 2013 Analysis of passively combined divided-pulse amplification as an energy-scaling concept Opt. Exp. 21 29031-42

Kienel M, Klenke A, Eidam T, Hädrich S, Limpert J and Tünnermann A 2014a Energy scaling of femtosecond amplifiers using actively controlled divided-pulse amplification Opt. Lett. 39 1049-52

Kienel M, Müller M, Demmler S, Rothhardt J, Klenke A, Eidam T, Limpert J and Tünnermann A 2014b Coherent beam combination of $\mathrm{Yb}$ :YAG single-crystal rod amplifiers $O p t$. Lett. 39 3278-81

Kienel M, Müller M, Klenke A, Eidam T, Limpert J and Tünnermann A 2015 Multidimensional coherent pulse addition of ultrashort laser pulses Opt. Lett. 40 522-5

Klenke A, Seise E, Limpert J and Tünnermann A 2011 Basic considerations on coherent combining of ultrashort laser pulses Opt. Express 19 25379-87

Klenke A, Kienel M, Eidam T, Hädrich S, Limpert J and Tünnermann A 2013 Divided-pulse nonlinear compression Opt. Lett. 38 4593-6

Klenke A, Hädrich S, Eidam T, Rothhardt J, Kienel M, Demmler S, Gottschall T, Limpert J and Tünnermann A 2014a 22 GW peak-power fiber chirped-pulse-amplification system Opt. Lett. 39 6875-8

Klenke A, Hädrich S, Kienel M, Eidam T, Limpert J and Tünnermann A 2014b Coherent combination of spectrally 
broadened femtosecond pulses for nonlinear compression $O p t$. Lett. 39 3520-2

Kong L J, Zhao L M, Lefrancois S, Ouzounov D G, Yang C X and Wise F W 2012 Generation of megawatt peak power picosecond pulses from a divided-pulse fiber amplifier $O p t$. Lett. 37 253-5

Krausst G, Lohss S, Hanke T, Sell A, Eggert S, Huber R and Leitenstorfer A 2010 Synthesis of a single cycle of light with compact erbium-doped fibre technology Nat. Photonics 4 33-6

Lamb E S, Wright L G and Wise F W 2014 Divided-pulse lasers Opt. Lett. 39 2775-7

Lombard L, Azarian A, Cadoret K, Bourdon P, Goular D, Canat G, Jolivet V, Jaouën Y and Vasseur O 2011 Coherent beam combination of narrow-linewidth $1.5 \mu \mathrm{m}$ fiber amplifiers in a long-pulse regime Opt. Lett. 36 523-5

Otto H-J, Klenke A, Jauregui C, Stutzki F, Limpert J and Tünnermann A 2014 Scaling the mode instability threshold with multicore fibers Opt. Lett. 39 2680-3

Papadopoulos D N, Friebel F, Pellegrina A, Hanna M, Camy P, Doualan J-L, Moncorgé R, Georges P and Druon F 2015 High repetition rate $\mathrm{Yb}: \mathrm{CaF} 2$ multipass amplifiers operating in the $100 \mathrm{~mJ}$ range J. Sel. Top. Quantum Electron. 213100211

Pogorelsky I, Agustsson R, Campese T, Murokh A, Ovodenko A, Polyanskiy M and Shaftan T 2014 High-repetition intra-cavity source of Compton radiation J. Phys. B: At. Mol. Opt. Phys. 47 234014

Potma E O, Evans C, Xie X S, Jones R J and Ye J 2003 Picosecondpulse amplification with an external passive optical cavity $O p t$. Lett. 28 1835-7

Pupeza I et al 2010 Power scaling of a high-repetition-rate enhancement cavity Opt. Lett. 35 2052-4

Pupeza I et al 2013 Compact high-repetition-rate source of coherent $100 \mathrm{eV}$ radiation Nat. Photon 7 608-12

Ramirez L P et al 2015 Coherent beam combining with an ultrafast multicore Yb-doped fiber amplifier Opt. Exp. 23 5406-16

Redmond S M, Ripin D J, Yu C X, Augst S J, Fan T Y, Thielen P A, Rothenberg J E and Goodno G D 2012 Diffractive coherent combining of a $2.5 \mathrm{~kW}$ fiber laser array into a $1.9 \mathrm{~kW}$ Gaussian beam Opt. Lett. 37 2832-4

Richardson D J, Nilsson J and Clarkson W A 2010 High power fiber lasers: current status and future perspectives J. Opt. Soc. Am. B 27 B63-92

Rigaud P, Kermene V, Bouwmans G, Bigot L, Desfarges-Berthelemot A, Labat D, Le Rouge A, Mansuryan T and Barthélémy A 2013 Spatially dispersive amplification in a 12-core fiber and femtosecond pulse synthesis by coherent spectral combining Opt. Exp. 21 $13555-63$
Seise E, Klenke A, Limpert J and Tünnermann A 2010 Coherent addition of fiber-amplified ultrashort laser pulses Opt. Exp. 18 27827-35

Shay T M, Benham V, Baker J T, Sanchez A D, Pilkington D and Lu C A 2007 Self-synchronous and self-referenced coherent beam combination for large optical arrays IEEE J. Sel. Top. Quantum Electron. 13 480-6

Shelton R K, Ma L-S, Kapteyn H C, Murnane M M, Hall J L and Ye J 2001 Phase-coherent optical pulse synthesis from separate femtosecond lasers Science 293 1286-9

Siiman L A, Chang W-Z, Zhou T and Galvanauskas A 2012 Coherent femtosecond pulse combining of multiple parallel chirped pulse fiber amplifiers Opt. Exp. 20 18097-116

Strickland D and Mourou G 1985 Compression of amplified chirped optical pulses Opt. Commun. 56219

Stutzki F, Jansen F, Otto H-J, Jauregui C, Limpert J and Tünnermann A 2014 Designing advanced very-large-modearea fibers for power scaling of fiber-laser systems Optica 1 233-42

Szatmari S and Simon P 1993 Interferometric multiplexing scheme for excimer amplifiers Opt. Comm. 98 181-92

Thielen P A et al 2012 Two-dimensional diffractive coherent combining of 15 fiber amplifiers into a $600 \mathrm{~W}$ beam Opt. Lett. 37 3741-3

Vorontsov M A and Sivokon V P 1998 Stochastic parallel-gradientdescent technique for high-resolution wave-front phasedistortion correction J. Opt. Soc. Am. A15 2745-58

Wang D, Leng Y and Huang Z 2014 Divided-pulse compression with gas-filled hollow-core fiber for generation of high-energy few-cycle pulses J. Opt. Soc. Am. B 31 1248-54

Weiss S B, Weber M E and Goodno G D 2012 Group delay locking of coherently combined broadband lasers Opt. Lett. 37 455-7

Zaouter Y, Daniault L, Hanna M, Papadopoulos D N, Morin F, Hönninger C, Druon F, Mottay E and Georges P 2012 Passive coherent combination of two ultrafast rod type fiber chirped pulse amplifiers Opt. Lett. 37 1460-2

Zaouter Y, Guichard F, Daniault L, Hanna M, Morin F, Hönninger C, Mottay E, Druon F and Georges P 2013 Femtosecond fiber chirped and divided pulse amplification system Opt. Lett. 38 106-8

Zapata-Rodríguez C J and Caballero M T 2007 Isotropic compensation of diffraction-driven angular dispersion $\mathrm{Opt}$. Lett. 32 2472-4

Zhou S, Wise F W and Ouzounov D G 2007 Divided-pulse amplification of ultrashort pulses Opt. Lett. 32 871-3

Zhou T, Ruppe J, Zhu C, Hu I-N, Nees J and Galvanauskas A 2015 Coherent pulse stacking amplification using low-finesse GiresTournois interferometers Opt. Exp. 23 7442-62 